Research Article

\title{
Deformation Analysis of Granular Soils under Dynamic Compaction Based on Stochastic Medium Theory
}

\author{
Jifang Du $\mathbb{D}^{1},{ }^{1}$ Shuaifeng $W u\left(\mathbb{D},{ }^{2}\right.$ Sen $\mathrm{Hou}^{3}$, and Yingqi $\mathrm{Wei}^{2}$ \\ ${ }^{1}$ Department of Transportation Science and Engineering, Beihang University, Beijing 100191, China \\ ${ }^{2}$ State Key Laboratory of Simulation and Regulation of Water Cycle in River Basin, China Institute of \\ Water Resources and Hydropower Research, Beijing 100048, China \\ ${ }^{3}$ The Engineering Research Base of China Airport Construction Corporation of CAAC, Beijing 100101, China
}

Correspondence should be addressed to Shuaifeng Wu; wusf@iwhr.com

Received 14 March 2019; Revised 2 June 2019; Accepted 16 June 2019; Published 2 July 2019

Academic Editor: Giovanni Garcea

Copyright (C) 2019 Jifang Du et al. This is an open access article distributed under the Creative Commons Attribution License, which permits unrestricted use, distribution, and reproduction in any medium, provided the original work is properly cited.

\begin{abstract}
Dynamic compaction (DC) is widely used to improve the mechanical properties of soils and other granular fill material upon which foundations or other structures are to be built. To calculate the inner deformation induced by DC, a computational model based on stochastic medium theory was developed to deduce the amount of deformation in the fill from the geometry of the DC crater. For this model, the tamper-soil system was simplified to an axisymmetric geometry and the probability of any unit of material below the crater being deformed can be calculated. Subsequently, the deformation at any point can be obtained by integration. Input parameters for the model were established by back analysis using the results from a DC field test and published data. Comparing the model results to results from actual and simulated DC programs shows that the computational model is useful for calculating deformation induced by DC without the need to consider the constitutive model of the soil.
\end{abstract}

\section{Introduction}

Compacting the soil that will be under a foundation is an essential procedure to ensure the stability of the structure. Improving the soil beneath the foundation can be accomplished by a number of different methods including static or dynamic rollers and fiber reinforcement [1-3]. However, static or dynamic rollers are limited and can only compact the soil to a relatively shallow depth $(0.2-1.0 \mathrm{~m})$ [4] and fiber reinforcement is expensive and not environment-friendly for large construction projects [5]. Compared with the above methods, dynamic compaction (DC) is a suitable method with the following advantages: (1) high density compaction to a considerable depth; (2) wide applicability in soils with different soil properties and different water contents; (3) simplicity of operation using widely available and simple equipment; (4) low cost. Menard [6] first introduced DC for construction and conducted systematically research on the method. Dynamic compaction simply involves dropping a tamper (10-40 t) from a height of 10-20 m onto the ground. Several blows are repeated until the ground under the tamper reaches the desired degree of soil improvement.

The extent of soil improvement is one of the most important concerns for a DC project; it is the essential indicator of the project's success. The soil improvement can be divided into two parts: the depth of improvement $(D I)$ and the lateral extent of improvement $(L I)$.

The $D I$ is a major issue facing the DC program designers and contractors [7]. The first equation for predicting DI was proposed by Menard [6] and it can be expressed as DI = $\sqrt{M H}$, where $M$ is the tamper weight in tones and $H$ is the falling height in meters. Mayne [8] summarized 120 sites with diverse soil properties and modified this equation to the form:

$$
D I=n \sqrt{M H}
$$

where $n$ (commonly equal to $0.3-0.8$ ) is an empirical constant related to soil properties. Subsequent researches by Leonards 
[9], Lukas [10], Rollins [11], Slocombe [12], and Luoguo [13] calibrated and determined different values for $n$ and proposed other forms for the equation based on the energy applied. However, these equations can only roughly evaluate the $D I$ and do not consider factors like soil properties and tamper radius. Although researchers like Chow [14] and Smits [15] proposed computational models that did consider these factors, the parameters in their models are difficult to measure, and the values these factors have affect the results.

The $L I$ is the maximum width the improvement extends horizontally away from the tamper. It is an essential parameter for determining the optimum distance between tamping points. There has been little research on how to calculate $L I$. Poran [16] discussed the factors that influence the degree of improvement and divided these factors into site-dependent factors and equipment-dependent factors. Two equations, one for the input energy and one for the dimensions of the plastic zone, were derived to evaluate the depth and width of improvement. The plastic zone was assumed to be semispherical shape.

In recent years, the dynamic mechanisms of DC have undergone further analysis and many simulations have been developed using finite or discrete element methods. For instance, Lee and $\mathrm{Gu}$ [17] proposed a method for estimating the degree and depth of improvement based on a two-dimensional finite element model. Ma [4] used the PFC2D/PFC3D software (Itasca Consulting Group, Inc., Minneapolis, MN, USA) to determine improvement and DI by analyzing porosity variations. Mostafa [18] conducted a comprehensive parametric study on the behavior of cohesive soils. Jia [19] investigated the behavior of granular soils during DC using PFC/FLAC software (Itasca Consulting Group). However, preparing these computational models and simulations is very complicated and time-consuming and it is not really feasible to use them in the field at construction sites.

During DC, the deformation can reflect the degree of soil improvement; this is a good indicator of how much the soil has been compacted. In most cases, when the soil in the crater is depressed less than a specified amounts per drop for the final two drops, it can be assumed that the soil's strength has been improved enough to meet the project's design requirements. The deformation in the soil can also be a reflection of the change in soil properties and used to determine how much the soil has been reinforced by the DC.

Stochastic medium theory was initially proposed by Litwiniszyn [20] and developed further by Liu et al. [21, 22]. The stochastic medium in question is granular material like sand, gravel, and rock fragments. The excavation of underground space often causes deformation of the overlying strata $[23,24]$ and the theory is mainly used to calculate subsidence after an opening has been excavated. The subsidence caused by the excavation will be transferred to the overlying strata (Figure 1(a)). For DC, the settling is caused by the impact of the tamper. Figure 1(b) shows soil particles compressed by tamping. Comparing Figure 1(a) with Figure 1(b) shows that the two kinds of settling are similar for noncohesive soils.

In this paper, the deformation caused by the DC is modeled using stochastic medium theory. A computational model is proposed based on this theory and two parameters, a compression factor (determined by the type of DC equipment used) and an influence angle (a function of the soil's properties). Finally, the applicability of this model is examined by comparing results calculated using the model with measurement made after a field test and with published data. This study can provide a method for determining how well a DC program is reinforcing the material upon which a foundation will be installed and will be valuable for the design and construction of DC projects.

\section{Model for Dynamic Compaction}

2.1. Introduction to Stochastic Medium Theory. In an Eulerian coordinate system, assume that the probability density for deformation of $d x$ is $f\left(x^{2}\right)$ for the $z$-axis symmetry of the deformation curve. The deformation probability $P$ for $d x$ can be expressed as

$$
P(d x)=f\left(x^{2}\right) d x .
$$

For an infinitesimal plane $d s=d x d y$ centered at point $A(x, y, z)$, as shown in Figure 2(a), the deformation probability is

$$
\begin{aligned}
P(d s) & =P(d x) P(d y)=f\left(x^{2}\right) d x f\left(y^{2}\right) d y \\
& =f\left(x^{2}\right) f\left(y^{2}\right) d s
\end{aligned}
$$

If the coordinate system $(x, y, z)$ is changed to $\left(x_{1}, y_{1}, z\right)$ as shown in Figure 2(b), then (3) is transformed into

$$
\begin{aligned}
P(d s) & =P\left(d x_{1}\right) P\left(d y_{1}\right)=f\left(x_{1}^{2}\right) d x f\left(y_{1}^{2}\right) d y \\
& =f\left(x_{1}^{2}\right) f\left(y_{1}^{2}\right) d s .
\end{aligned}
$$

If the $x_{1}$ axis passes through the point $A$, then

$$
\begin{aligned}
x_{1}^{2} & =x^{2}+y^{2}, \\
y_{1} & =0 \\
f\left(x_{1}^{2}\right) f\left(y_{1}^{2}\right) d s & =f\left(x^{2}+y^{2}\right) f(0) d s \\
& =c f\left(x^{2}+y^{2}\right) d s \\
f\left(x^{2}\right) \cdot f\left(y^{2}\right) & =f\left(x_{1}^{2}\right) f\left(y_{1}^{2}\right)=c \cdot f\left(x^{2}+y^{2}\right)
\end{aligned}
$$

in which $c$ is a constant.

The derivation of (7) will yield:

$$
\begin{aligned}
f\left(y^{2}\right) \frac{\partial f\left(x^{2}\right)}{\partial x^{2}} & =c \frac{\partial f\left(x^{2}+y^{2}\right)}{\partial\left(x^{2}+y^{2}\right)} \frac{\partial\left(x^{2}+y^{2}\right)}{\partial x^{2}} \\
& =c \frac{\partial f\left(x^{2}+y^{2}\right)}{\partial\left(x^{2}+y^{2}\right)} \\
f\left(x^{2}\right) \frac{\partial f\left(y^{2}\right)}{\partial y^{2}} & =c \frac{\partial f\left(x^{2}+y^{2}\right)}{\partial\left(x^{2}+y^{2}\right)} \frac{\partial\left(x^{2}+y^{2}\right)}{\partial y^{2}} \\
& =c \frac{\partial f\left(x^{2}+y^{2}\right)}{\partial\left(x^{2}+y^{2}\right)} ;
\end{aligned}
$$




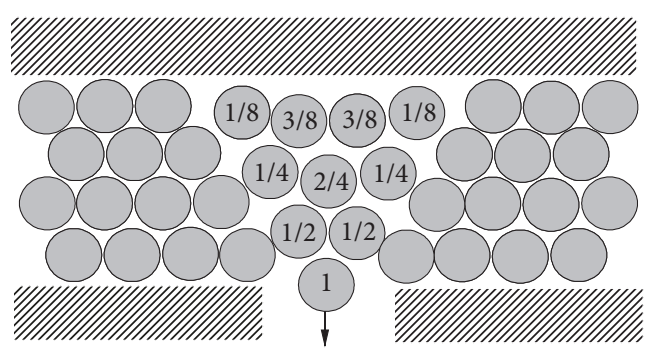

(a) Mining subsidence

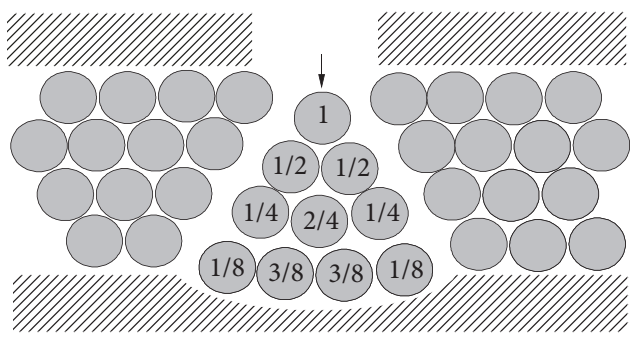

(b) Dynamic compaction

FIGURE 1: Sketches showing the deformation induced by (a) mining subsidence and (b) dynamic compaction.

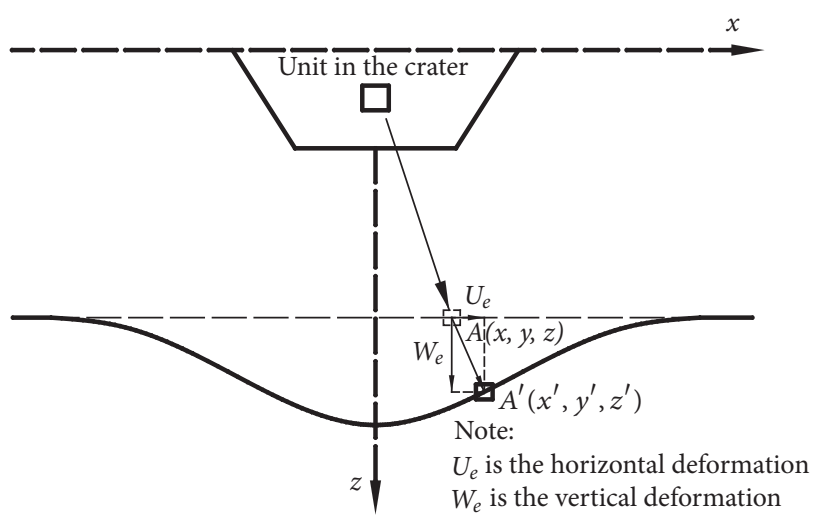

(a) Deformation of point $A$

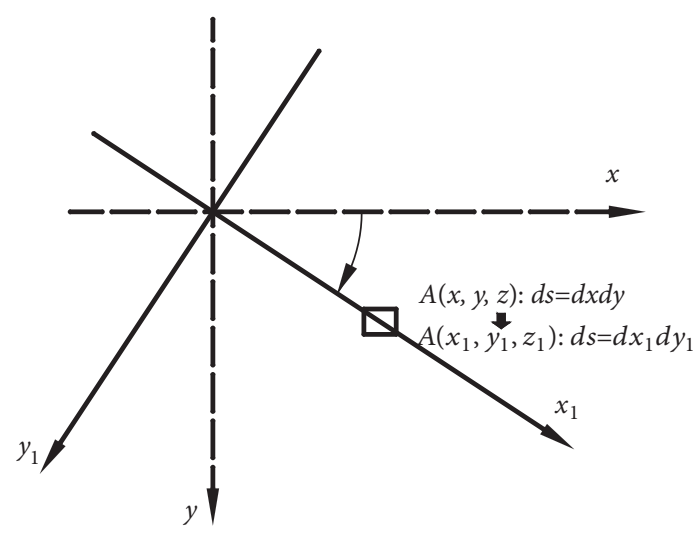

(b) The transformation of coordinate system

FIGURE 2: Diagrams showing the deformation of point $A$ in a coordinate system and in a transformed coordinate system for the DC calculation model.

therefore

$$
\begin{aligned}
f\left(y^{2}\right) \frac{\partial f\left(x^{2}\right)}{\partial x^{2}} & =f\left(x^{2}\right) \frac{\partial f\left(y^{2}\right)}{\partial y^{2}} \\
\text { or } \frac{1}{f\left(x^{2}\right)} \frac{\partial f\left(x^{2}\right)}{\partial x^{2}} & =\frac{1}{f\left(y^{2}\right)} \frac{\partial f\left(y^{2}\right)}{\partial y^{2}} .
\end{aligned}
$$

Equation (9) shows that the two sides of the equation are independent of $x$ and $y$.

Let

$$
\frac{1}{f\left(x^{2}\right)} \frac{\partial f\left(x^{2}\right)}{\partial x^{2}}=k_{1}{ }^{2}
$$

Then, after integration, the following equation can be obtained:

$$
f\left(x^{2}\right)=k_{2} e^{-k_{1}^{2} x^{2}}
$$

where $k_{1}$ and $k_{2}$ are parameters corresponding to the depth, $z$.

Therefore, the deformation probability of plane $d s$ is

$$
P(d s)=f\left(x^{2}\right) f\left(y^{2}\right) d x d y=k_{2}^{2} e^{-k_{1}^{2}\left(x^{2}+y^{2}\right)} d x d y .
$$

Assuming that the soil is not compacted, the deformation should be equal to the volume of the unit $\left(V_{e}\right)$ in the crater, so

$$
V_{e}=\int_{-\infty}^{+\infty} \int_{-\infty}^{+\infty} f\left(x^{2}\right) f\left(y^{2}\right) d x d y=1
$$

After solving, it can be seen that

$$
k_{2}^{2}=\frac{\pi}{k_{1}^{2}} \text {. }
$$

Based on stochastic medium theory, the parameter $k_{1}$ can be expressed as $r(z)$ (also called the influence radius) and $r(z)$ is defined as

$$
r(z)=\frac{z}{\tan \beta}
$$

where $z$ is the depth of $d s$ and $\beta$ is the influence angle.

Therefore, the deformation at the point $(x, y, z)$ induced by a unit in the crater can be expressed as

$$
W_{e}=\frac{1}{r^{2}(z)} \exp \frac{-\pi\left(x^{2}+y^{2}\right)}{r^{2}(z)} \text {. }
$$


2.2. Computational Model. Assuming that the foundation surface is smooth and the face of the tamper is circular, the crater formed by the tamper can be simplified as a frustum of a cone (Figure 3) and the DC can be simplified into an axisymmetric problem. To simplify the calculation, we only study the deformation in plane $z o x$ of the coordinate system. The deformation of point $A$ induced by the unit in the crater can be divided into two directions: the vertical and the horizontal, which are $W_{e}$ and $U_{\mathrm{e}}$ in Figure 2(a), respectively. The vertical deformation is much easier to be detected than the horizontal. Therefore, in this paper, only vertical displacement is considered; horizontal displacement is ignored. Taking the central axis of the crater as the $z$-axis, for a unit $d s=d x_{e} d y_{e} d z_{e}$ centered at $\left(x_{e}, y_{e}, z_{e}\right)$ in the crater, the induced deformation at point $A(x, y, z)$ (Figure 2) can be expressed by

$$
\begin{aligned}
W_{e}= & \frac{1}{r^{2}\left(z-z_{e}\right)} \\
& \cdot \exp \frac{-\pi\left[\left(x-x_{e}\right)^{2}+\left(y-y_{e}\right)^{2}\right]}{r^{2}\left(z-z_{e}\right)} d x_{e} d y_{e} d z_{e} .
\end{aligned}
$$

The deformation induced in the crater can be obtained by integration, so

$$
\begin{aligned}
& W(x, y, z) \\
& \quad=\iiint_{\Omega} \frac{1}{r^{2}\left(z-z_{e}\right)} \exp \frac{-\pi\left[\left(x-x_{e}\right)^{2}+\left(y-y_{e}\right)^{2}\right]}{r^{2}\left(z-z_{e}\right)} d x_{e} d y_{e} d z_{e}
\end{aligned}
$$

where $\Omega$ represents the whole region of the crater.

The crater's coordinate system can be transformed into cylindrical coordinates and the unit in the crater changed from $d x_{e} d y_{e} d z_{e}$ to $\rho d \theta d \rho d \zeta$. The geometric parameters of the crater (Figure 3 ) are the depth, $h$, and the depth within the $(0, h)$ range represented by $\zeta$; a vertical section through the crater is trapezoidal and the radius at different crater depths can be expressed as $\rho(\zeta)$ which can be calculated from

$$
\rho(\zeta)=\rho_{1}+\frac{\left(\rho_{2}-\rho_{1}\right) \zeta}{h}
$$

where $\rho_{1}$ and $\rho_{2}$ are the crater's top and the bottom radii.

The deformation at any depth $z$ can be calculated from

$$
\begin{aligned}
& W(x, z) \\
& =\eta \int_{0}^{h} \int_{0}^{\rho(\zeta)} \int_{0}^{2 \pi} \frac{1}{r^{2}(z-\zeta)} \exp \left(-\pi \frac{(x-\rho \cos \theta)^{2}+(0-\rho \sin \theta)^{2}}{r^{2}(z-\zeta)}\right) \\
& \cdot \rho d \theta d \rho d \zeta \\
& =\eta \int_{0}^{h} \int_{0}^{\rho(\xi)} \int_{0}^{2 \pi} \frac{\tan ^{2} \beta}{(z-\zeta)^{2}} \exp \left(-\pi \tan ^{2} \beta \frac{(x-\rho \cos \theta)^{2}+(\rho \sin \theta)^{2}}{(z-\zeta)^{2}}\right) \\
& \cdot \rho d \theta d \rho d \zeta
\end{aligned}
$$

Dynamic compaction makes the soil denser. Therefore, a compression coefficient, $\eta$, must be introduced to calculate the vertical movement of a unit of compacted material.

The influence angle $\beta$ can reflect the properties of the soil at depth $z$. However, if the soil at the construction site is considered to be homogeneous, the influence angle will be constant. For DC, the impact energy influence angle is the main factor affecting the amount of compaction. Therefore, the impact energy influence angle $\beta$ is used in the calculations in this paper. According to Yao [25], the DC impact influence angle for gravelly soil can be assumed to be $30^{\circ}$ from the horizontal, so $30^{\circ}$ is used as the influence angle in all the calculations presented below. The subsidence at a specific depth can be calculated using (20); the only unknown is $\eta$, the compression coefficient.

\section{Model Verification}

3.1. Field Test Results. To acquire values for the variables in the model, a field test was conducted at the Chengde airport construction site, Hebei Province, China. The construction material was mainly crushed rock that complied with airport fill technical code MH/T5035-2017 [26]. Particle gradation curves for the eight different 1-m layers in the fill are shown in Figure 4.

The machine used for the DC was a 70-t crawler. The tamper weighed $16.8 \mathrm{t}$ and was $2.5 \mathrm{~m}$ in diameter. The tamper imparted a DC energy of $1000 \mathrm{kNm}$ with a fall of $6 \mathrm{~m}$. To show the amount of settling in the different fill layers caused by the DC, thin plastic sheeting was laid down at three different horizons as the fill was being placed. The sheeting was installed at depths of $1.85 \mathrm{~m}, 2.8 \mathrm{~m}$, and $4 \mathrm{~m}$ below the final fill surface as shown in Figures 5 and 6 . For the calculations below, the geometry of the crater was simplified to make the crater a cylinder with a radius of $1.2 \mathrm{~m}$ and the parameters used in the calculations were $\rho_{1}=\rho_{2}=1.2 \mathrm{~m}, h=$ $0.8 \mathrm{~m}$.

The most appropriate value of $\eta$ is the lowest least squares value for $\eta$ calculated using

$$
R(\eta)=\sum\left(W_{i}-W_{i}^{*}\right)^{2}
$$

where $W_{i}$ and $W_{i}^{*}$ are the calculated and observed results for deformation, respectively. The lower the $R(\eta)$, the better the match between the measured and the calculated result.

The observed and the calculated data are listed in Table 1. The calculated values are obviously larger than the observed values because the calculated values do not take the compression coefficient into account. The relationship between $R(\eta)$ and $\eta$ was calculated using (21) and the results are shown in Figure 7. As shown in the figure, $R(\eta)$ is minimized when $\eta$ $=0.65$. The modified data listed in Table 1 were calculated with $\eta=0.65$. The final deformation is shown in Figure 8; the modified values agreed well with the observed values.

3.2. Published Data (Ghassemi et al., [27]). The below-surface deformation induced by DC is rarely reported in field test results. Therefore, the results from this study are compared with the results from a DC simulation published by Ghassemi et al. [27]. Ghassemi et al. used finite element software to simulate sand compaction and verified the results of Oshima and Takada's [28] centrifuge tests. The simulated DC energy was $4000 \mathrm{kNm}$, energy equivalent to a 20 - $\mathrm{t}$ tamper dropping $20 \mathrm{~m}$. Figure 1 in [27] shows the deformation after DC. The 
TABLE 1: Calclulated, observed, and modified deformation for the fill at the three horizons covered by plastic sheeting at the field test site at different horizontal distances from the tamper (unit: $\mathrm{cm}$ ).

\begin{tabular}{|c|c|c|c|c|c|c|c|}
\hline \multirow{2}{*}{ Depth } & & \multicolumn{6}{|c|}{ Horizontal distance } \\
\hline & & $0 \mathrm{~m}$ & $1 \mathrm{~m}$ & $2 \mathrm{~m}$ & $3 \mathrm{~m}$ & $4 \mathrm{~m}$ & $5 \mathrm{~m}$ \\
\hline \multirow{3}{*}{$1.85 \mathrm{~m}$} & Calculated & 42 & 29.27 & 9.62 & 1.59 & 0.156 & 0.001 \\
\hline & Observed & 25.5 & 20.5 & 10 & 5 & 0 & 0 \\
\hline & Modified & 27.3 & 19.03 & 6.25 & 1.03 & 0.1 & 0 \\
\hline \multirow{3}{*}{$2.8 \mathrm{~m}$} & Calculated & 18.8 & 15.9 & 9.69 & 4.29 & 1.403 & 0.349 \\
\hline & Observed & 15.5 & 15.5 & 8 & 3 & 1 & 0 \\
\hline & Modified & 12.22 & 10.34 & 6.3 & 2.79 & 0.9 & 0.23 \\
\hline \multirow{3}{*}{$4 \mathrm{~m}$} & Calculated & 8.88 & 8.215 & 6.502 & 4.41 & 2.57 & 1.29 \\
\hline & Observed & 6 & 6 & 4 & 2.5 & 1.5 & 0 \\
\hline & Modified & 5.8 & 5.4 & 4.2 & 2.9 & 1.67 & 0.84 \\
\hline
\end{tabular}

Note: Modified value $=$ Calculated value $\times$ Compression Coefficient

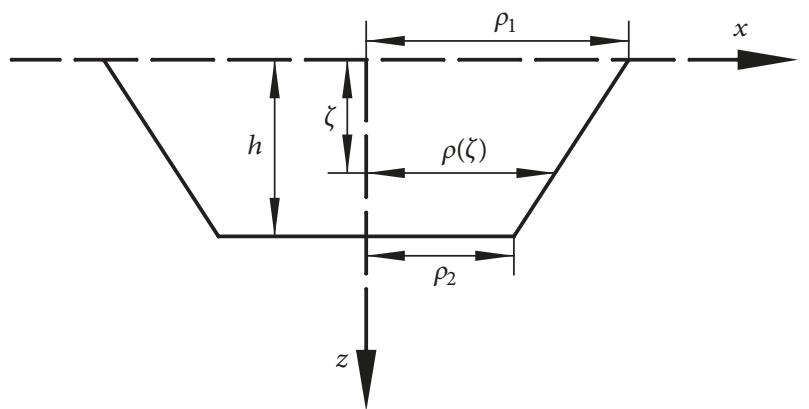

FIGURE 3: Cross-section showing the idealized geometry of the crater created by a circular tamper during dynamic compaction.

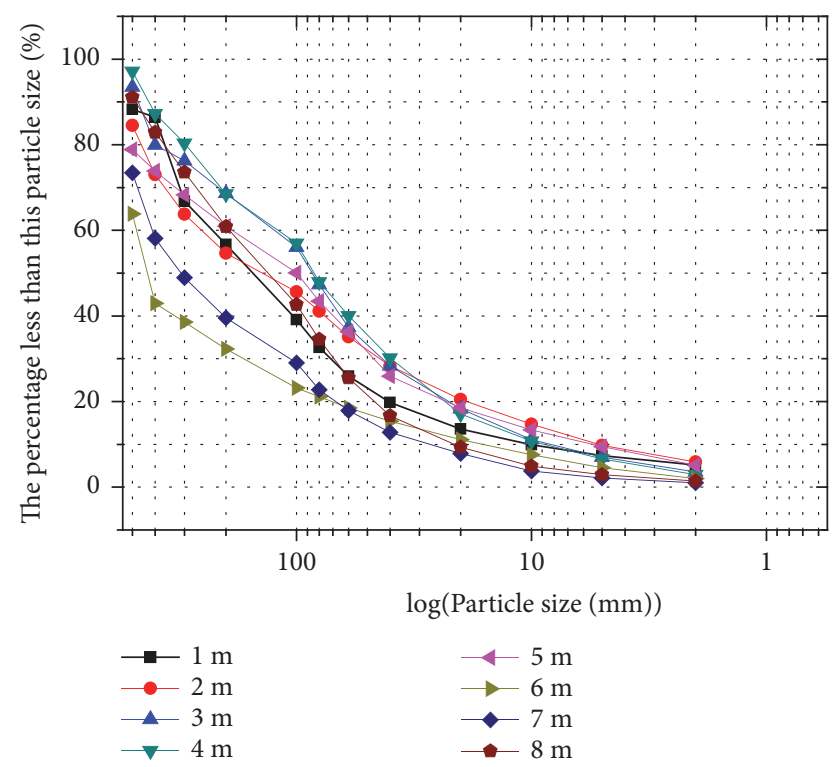

FIgURE 4: Particle gradation curves for the 1-m layers in the fill at the Chengde airport construction site used for the field DC test.

dimensions of the simulated crater in Ghassemi et al. were $\rho_{1}=1.4 \mathrm{~m}, \rho_{2}=1.2 \mathrm{~m}, h=1.2 \mathrm{~m}$. Those crater dimensions were entered into (20) to calculate the soil deformation for that tamper configuration; the results are listed in Table 2.

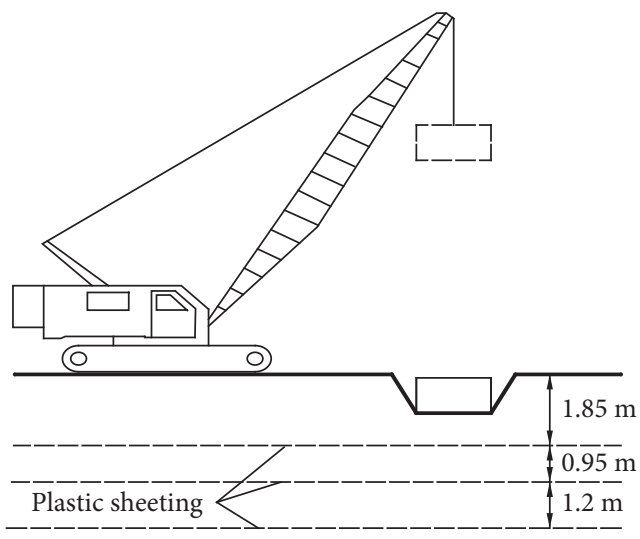

FIGURE 5: Sketch showing the layout of the field test and the positions of the plastic sheeting used to monitor the amount of fill settling.

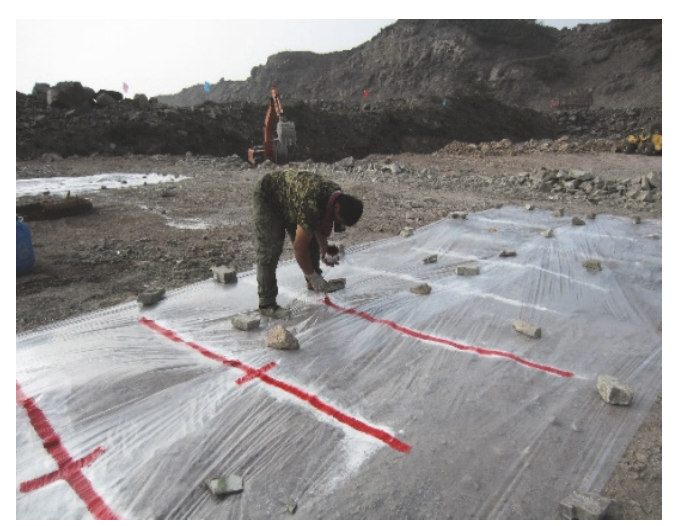

FIGURE 6: A worker laying out plastic sheeting on the top of a 1-m fill layer that will be buried by subsequent layers of fill.

Figure 9 shows that $\eta$ for this DC simulated configuration is 0.7 , so that value was used to calculate the "Modified" results listed in Table 2. As shown in Figure 10, the modified results show a good correlation with the data from Ghassemi et al. 


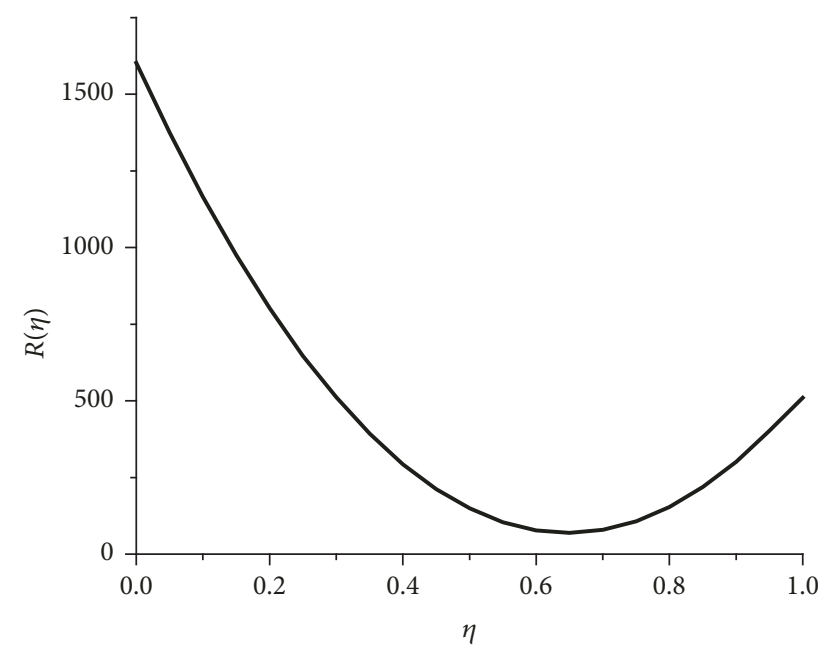

FIGURE 7: Graph showing the relationship between the compression factor, $\eta$, and the least square variance between observed and calculated results, $R(\eta)$, for the field test data.

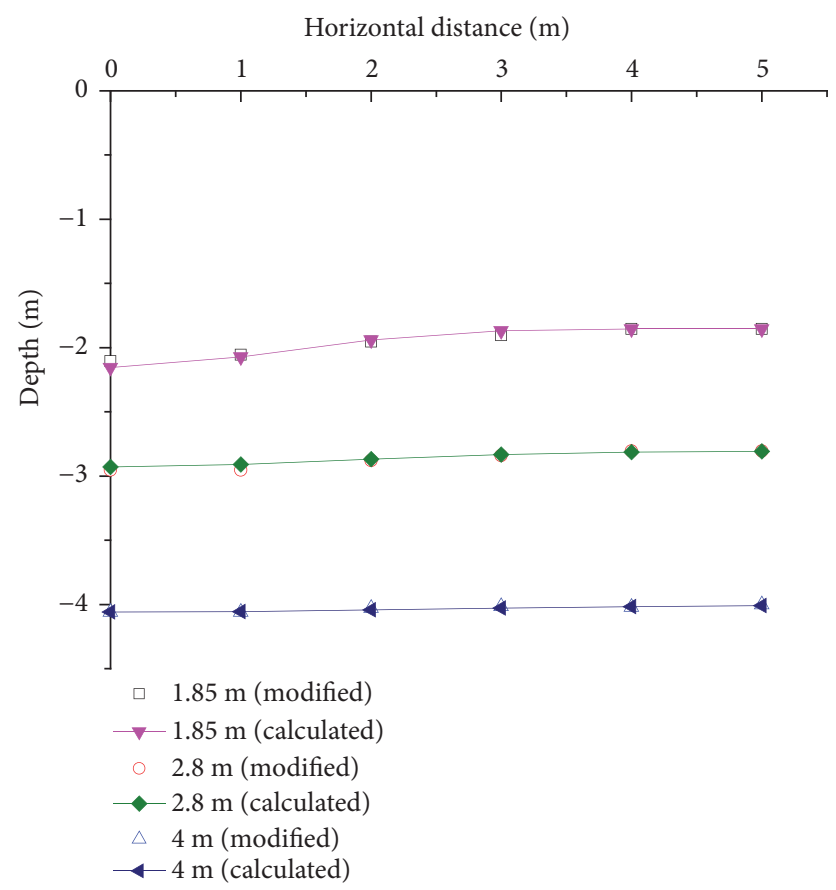

FIGURE 8: Cross-section showing the upper $4 \mathrm{~m}$ of the fill (the center of the DC crater is at horizontal position " 0 ") and the observed and modified results at different depth.

\section{Discussion}

4.1. Critical Deformation. The computational model has shown its applicability for DC analysis. However, the primary shortcoming of this model is that the calculated deformation cannot be zero even for locations far removed from the tamped crater. This means that the zero deformation boundary cannot be determined using this model. To compensate for this shortcoming, the concept of a critical level of deformation has been formulated. The critical level of deformation is defined as deformation sufficient to significantly reinforce

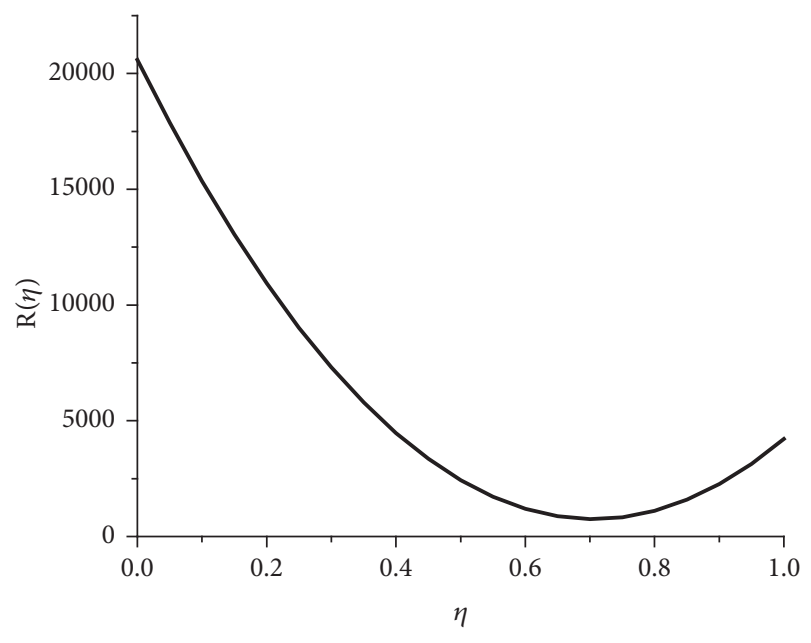

FIGURE 9: Graph showing the relationship between the compression factor, $\eta$, and the least square variance between observed and calculated results, $R(\eta)$, for the data published by Ghassemi et al. [27].

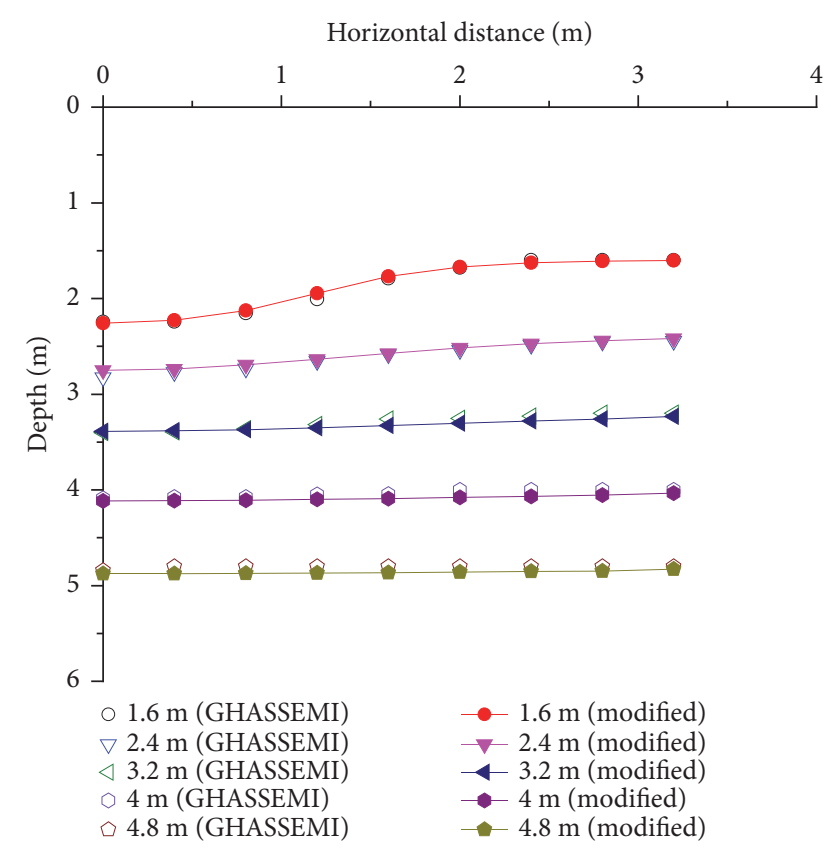

FIGURE 10: Graph comparing the deformation values from Ghassemi et al.s [27] simulated DC on sand to deformation values calculated from the dimensions of Ghassemi's crater using the calculation model developed for this study.

the soil. Deformation below this level will just modify the soil but not increase its strength. By comparing the Standard penetration test $N_{120}$ (SPT) values from the fill at the Chengde airport construction site before and after DC (Figure 11), the rate of improvement can be calculated. Equation (22) can be used to quantify the amount of improvement.

$$
\omega=\frac{S P T \text { after DC }-S P T \text { before DC }}{S P T \text { before DC }} \times 100 \%
$$

where $\omega$ is the rate of improvement. 
TABLE 2: Calculated, observed, and modified deformations for simulated sand fill. Observed data are from Ghassemi et al. [27]; calculated and modified data are from the computational model applied to Ghassemi's data (unit: $\mathrm{cm}$ ).

\begin{tabular}{|c|c|c|c|c|c|c|c|c|c|c|}
\hline \multirow{2}{*}{ Depth } & & \multicolumn{9}{|c|}{ Horizontal distance } \\
\hline & & $0 \mathrm{~m}$ & $0.4 \mathrm{~m}$ & $0.8 \mathrm{~m}$ & $1.2 \mathrm{~m}$ & $1.6 \mathrm{~m}$ & $2 \mathrm{~m}$ & $2.4 \mathrm{~m}$ & $2.8 \mathrm{~m}$ & $3.2 \mathrm{~m}$ \\
\hline \multirow{3}{*}{$1.6 \mathrm{~m}$} & Calculated & 94.1 & 89.7 & 75 & 49.3 & 24.1 & 10.2 & 4 & 1.58 & 0.48 \\
\hline & Observed & 64.6 & 64 & 55.1 & 40.8 & 18.9 & 7.7 & 0 & 0 & 0 \\
\hline & Modified & 65.8 & 62.8 & 52.5 & 34.5 & 16.9 & 7.14 & 2.8 & 1.1 & 0.33 \\
\hline \multirow{3}{*}{$2.4 \mathrm{~m}$} & Calculated & 50.3 & 48.1 & 42 & 33.6 & 24.6 & 16.6 & 10.4 & 6.1 & 2.6 \\
\hline & Observed & 42.5 & 37 & 33.4 & 25.4 & 18.3 & 13.9 & 8.6 & 5.3 & 4.6 \\
\hline & Modified & 35.2 & 33.6 & 29.4 & 23.5 & 17.3 & 11.6 & 7.27 & 4.26 & 1.84 \\
\hline \multirow{3}{*}{$3.2 \mathrm{~m}$} & Calculated & 26.8 & 26.2 & 24.4 & 21.6 & 18.3 & 14.8 & 11.5 & 8.5 & 4.55 \\
\hline & Observed & 20.3 & 19.7 & 16.1 & 11.8 & 5.9 & 5.39 & 3 & 0 & 0 \\
\hline & Modified & 18.8 & 18.3 & 17.1 & 15.1 & 12.8 & 10.4 & 8 & 6 & 3.2 \\
\hline \multirow{3}{*}{$4 \mathrm{~m}$} & Calculated & 16.26 & 16 & 15.35 & 14.3 & 12.9 & 11.4 & 9.7 & 8 & 4.7 \\
\hline & Observed & 8.9 & 7.6 & 7.6 & 4.8 & 4.4 & 0 & 0 & 0 & 0 \\
\hline & Modified & 11.4 & 11.2 & 10.7 & 10 & 9.06 & 8 & 6.8 & 5.6 & 3.3 \\
\hline \multirow{3}{*}{$4.8 \mathrm{~m}$} & Calculated & 10.8 & 10.7 & 10.4 & 9.94 & 9.3 & 8.55 & 7.7 & 6.8 & 4.1 \\
\hline & Observed & 7 & 6 & 6 & 0 & 0 & 0 & 0 & 0 & 0 \\
\hline & Modified & 7.56 & 7.5 & 7.3 & 6.9 & 6.5 & 6 & 5.4 & 4.8 & 2.9 \\
\hline
\end{tabular}

Note: Modified value $=$ Calculated value $\times$ Compression Coefficient

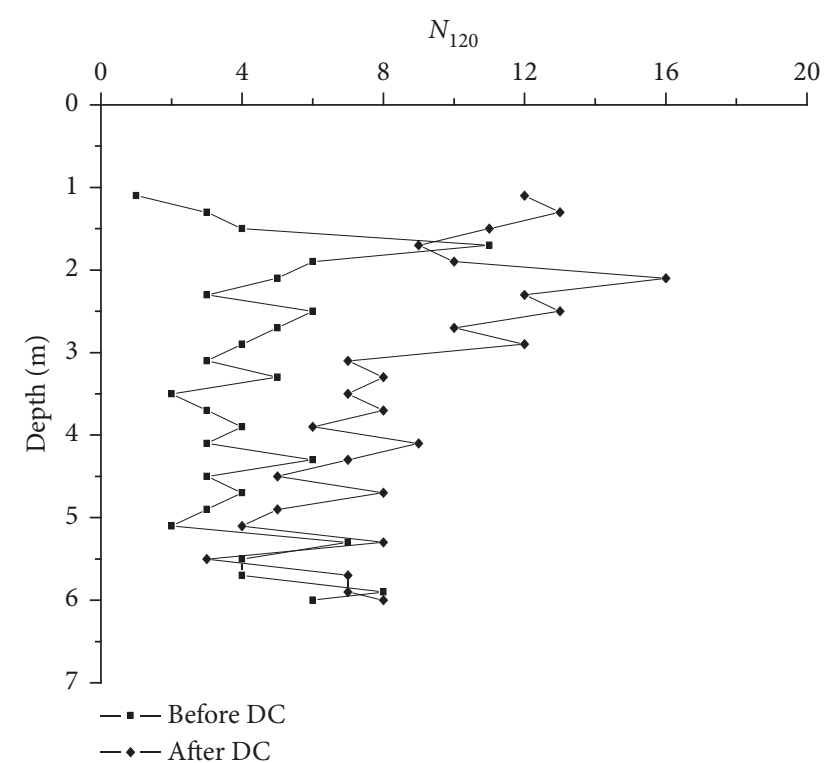

Figure 11: Dynamic cone penetration test results $\left(N_{120}\right)$ from the fill at the Chengde airport construction site before and after DC.

Figure 12 is a graph showing the rate of improvement for a number of points in the fill plotted against the corresponding inner deformation. The fitted equation for the curve on that figure is

$$
W=0.28 e^{\omega / 0.65}+3.5
$$

According to SPT's performed at the construction site, the DI where the rate of improvement is $50 \%$ is about $5 \mathrm{~m}$. The vertical deformation of points at the $5-\mathrm{m}$ depth, from (22), is $0.04 \mathrm{~m}$. Therefore, $0.04 \mathrm{~m}$ was determined to be the amount

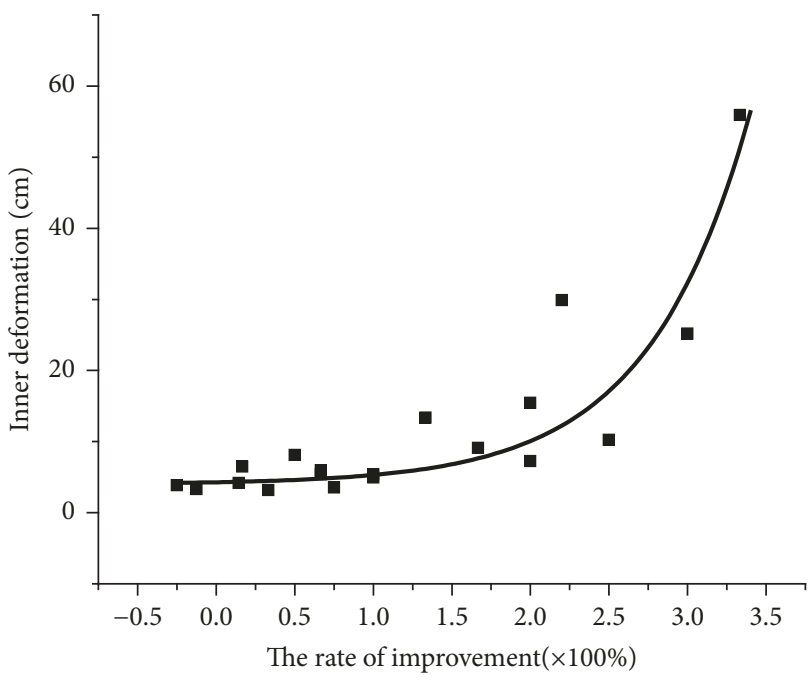

FIGURE 12: Graph showing the percentage of SPT penetration improvement for several points in the Chengde airport construction site fill after DC.

of deformation that defines the critical level of deformation boundary. Points within that boundary should have had their strength improved by at least $50 \%$. The boundary's location is defined by points with a vertical deformation of $0.04 \mathrm{~m}$; a line drawn thorough those points is shown in Figure 13. The line shows that the reinforced area is bubble-shaped and the reinforced area extends nearly $3 \mathrm{~m}$ from the center of the crater. The area of reinforcement is consisted with the field test results.

4.2. Crater Depth and the Reinforcement Extent. The DI and $L I$ away from the tamping sites are two essential parameters that must be considered when a DC program is being 


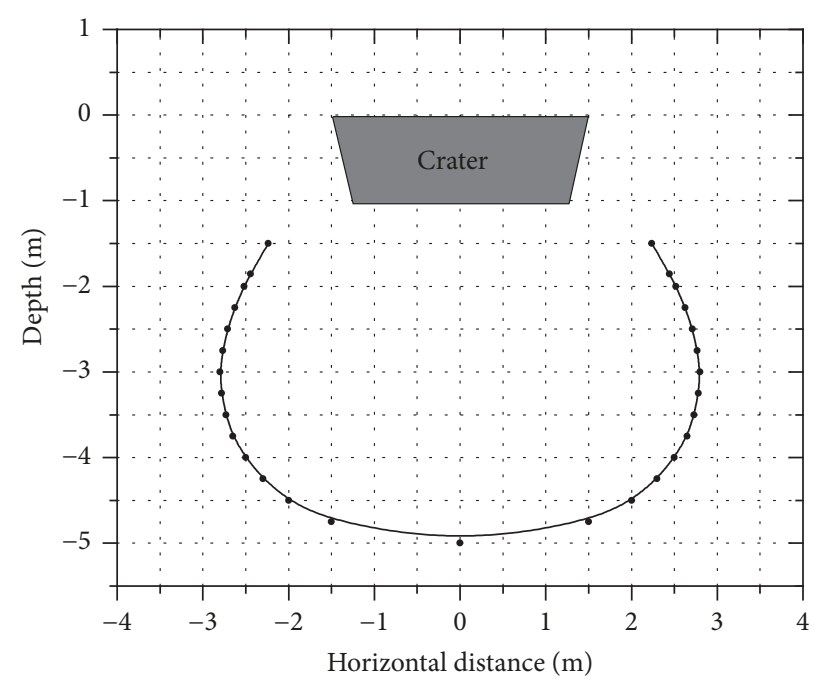

FIGURE 13: Cross-section showing the "critical level of deformation" boundary, the line within which deformation exceeds $0.04 \mathrm{~m}$.

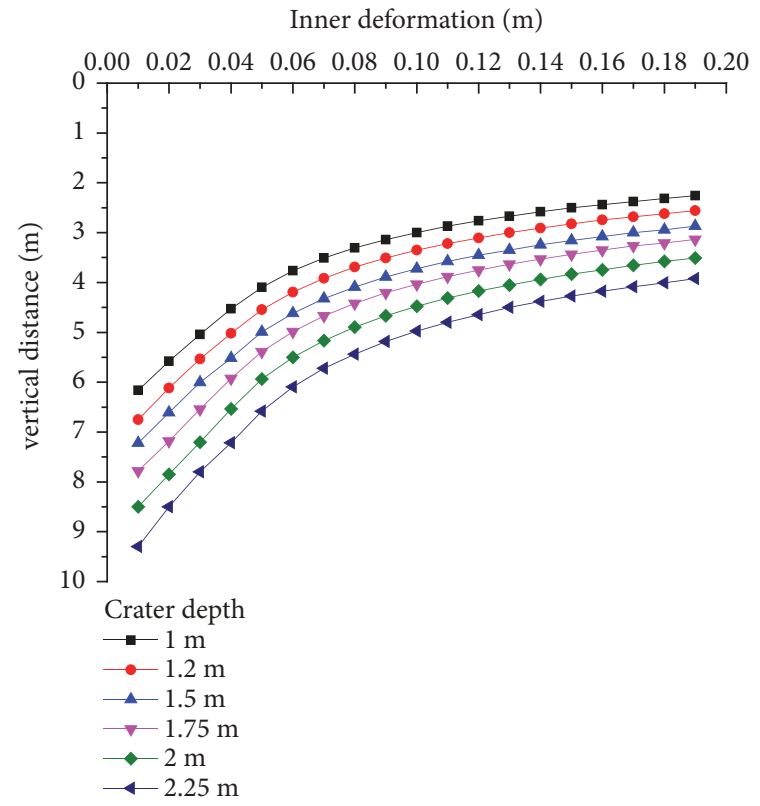

(a) Vertical direction

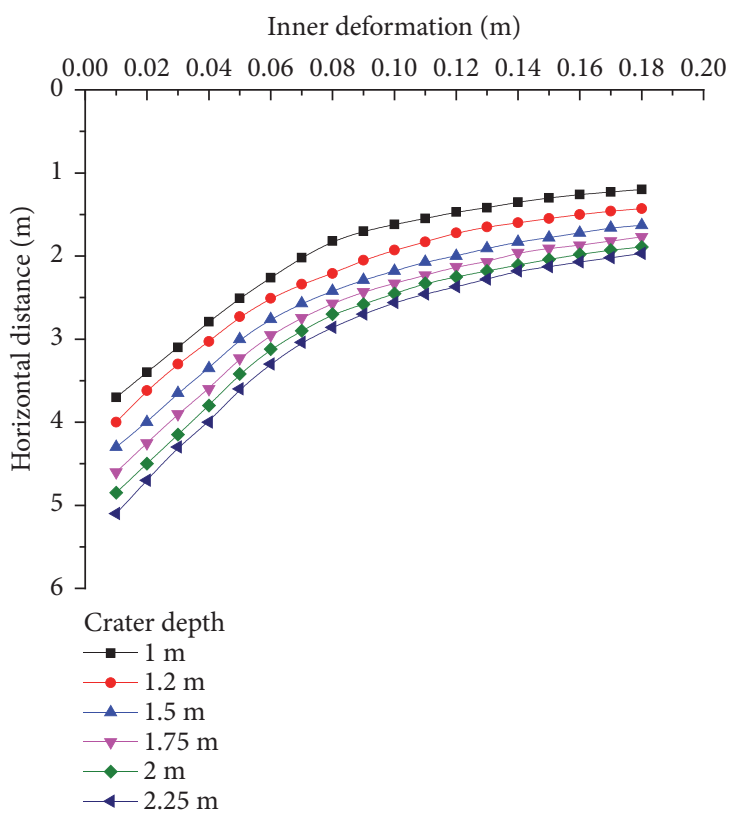

(b) Horizontal direction

FIGURE 14: The change of inner deformation with different crater depth along the (a) vertical and (b) horizontal direction.

designed. These parameters are commonly determined by using the Menard equation or practical field experience; both are largely based on the energy produced by the DC. However, for soils with different properties, the same impact energy produces different degrees of reinforcement. Because it is difficult to observe the change that have taken place in the soil after DC, the tamper-soil system is somewhat of a black box in that we only know the inputs to the system but cannot easily monitor the results. However, an "external interface" for this "black box" exists on the geometry of the crater. For the same DC energy, the shapes and the depths of craters are different for different soils. The crater is a reflection of the soil-tamper system and can be used to analyze the posttamping changes in the soil. In our stochastic-mediumbased computational model, the crater is used as the main indicator of soil reinforcement. Figure 14 is the deformation of different depth with different crater depth calculated by the model. The deformation is decreased with the increase of the depth and radial distance. Meanwhile, the increase of the crater depth is corresponding to a larger reinforcement extent.

Figures 15(a) and 15(b) present the relationships between $D I$ and crater depth, $L I$ and crater depth, respectively. The $D I$ increases with the growth of the crater depth and the correlation is very nearly linear. The $L I$ shows the same character with the DI, but the trend of growth slows down 


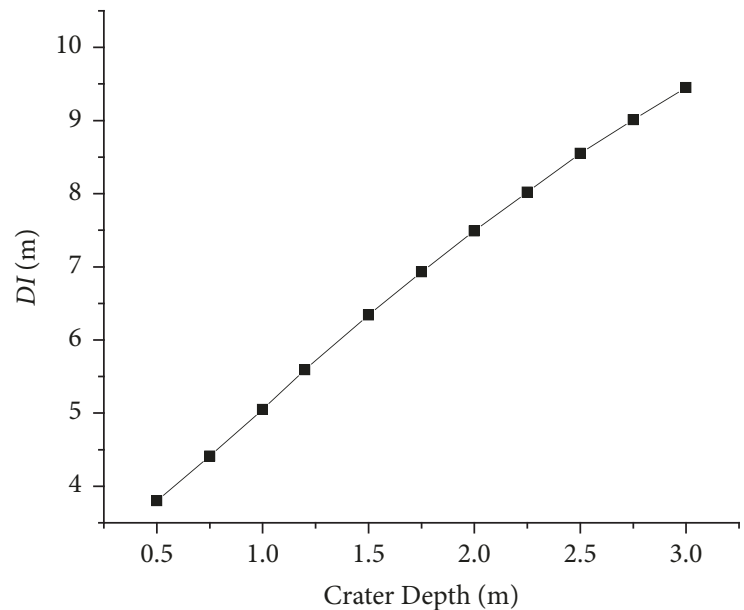

(a) $D I$

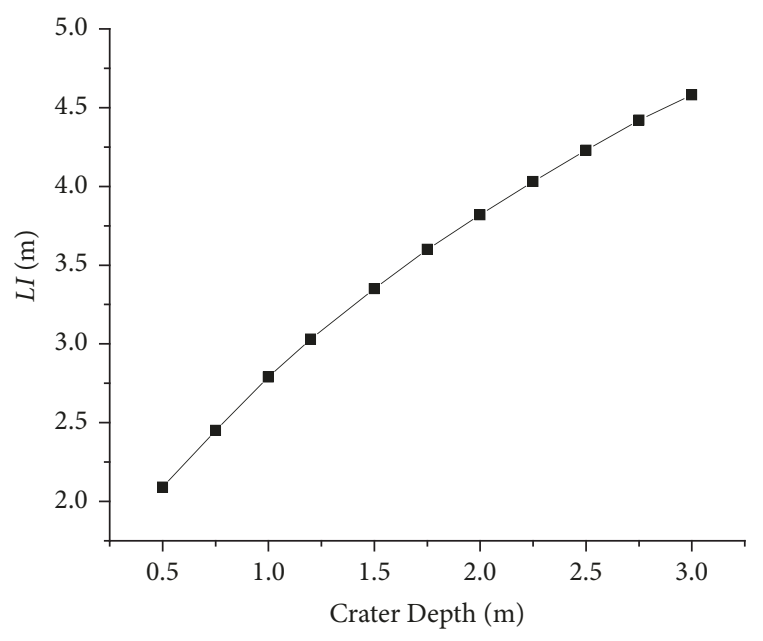

(b) $L I$

FIGURE 15: The relationship of crater depth and (a) DI, depth of soil improvement, and (b) $L I$, lateral extent of soil improvement.

with the increase of crater depth. When the crater depth increases from $1 \mathrm{~m}$ to $2 \mathrm{~m}$, the growth of $L I$ is about 1.2 $\mathrm{m}$. However, the growth of $L I$ is just $0.6 \mathrm{~m}$ when the crater depth increases from $2 \mathrm{~m}$ to $3 \mathrm{~m}$. Therefore, it can be concluded that, increasing the crater depth, the DI can be significantly expanded while $L I$ increases consistently with a slowly decreasing rate (Figure 15(b)).

\section{Conclusions}

A computational model based on stochastic medium theory has been developed to determine how much dynamic compaction (DC) reinforces the soil. This model is verified by comparing model results with the results from two compaction programs, one on crushed rock fill and the other simulating a sand fill. The compression coefficients for these two programs are 0.65 for the crushed rock and 0.7 for the sand. This method for establishing the degree of reinforcement has several advantages when compared with traditional methods. First, it takes the geometry of the crater into account. The shape and depth of the crater can reflect the DC energies and the soil properties and the crater's dimension are easy to obtain at the construction site with no special equipment or personnel training required. Second, the model assesses the extent of the reinforcement both laterally and vertically instead of merely estimating the $D I$. Finally, the computation is simple and can be easily solved using commercially available programs. However, this model is a prototype and more details should be studied. Additional validation of the computational model is also needed before sufficient confidence on the model's results can be established.

\section{Data Availability}

The data used to support the findings of this study are available from the corresponding author upon request.

\section{Conflicts of Interest}

The authors declare no conflicts of interest.

\section{Acknowledgments}

This research was supported by the National Basic Research Program of China, grant number 2014CB047004. We thank David Frishman, Ph.D0, from Liwen Bianji, Edanz Group, China, for editing the English text of a draft of this manuscript.

\section{References}

[1] Y. Wang, P. Guo, F. Dai, X. Li, Y. Zhao, and Y. Liu, "Behavior and modeling of fiber-reinforced clay under triaxial compression by combining the superposition method with the energy-based homogenization technique," International Journal of Geomechanics, vol. 18, no. 12, Article ID 04018172, 2018.

[2] Y. Wang, P. Guo, X. Li, H. Lin, Y. Liu, and H. Yuan, "Behavior of fiber-reinforced and lime-stabilized clayey soil in triaxial tests," Applied Sciences, vol. 9, no. 5, Article ID 900, 2019.

[3] Y. X. Wang, P. P. Guo, H. Lin et al., "Numerical analysis of fiber-reinforced soils based on the equivalent additional stress concept," International Journal of Geomechanics, 2019.

[4] Z. Ma, F. Dang, and H. Liao, "Numerical study of the dynamic compaction of gravel soil ground using the discrete element method," Granular Matter, vol. 16, no. 6, pp. 881-889, 2014.

[5] S.-J. Feng, K. Tan, W.-H. Shui, and Y. Zhang, "Densification of desert sands by high energy dynamic compaction," Engineering Geology, vol. 157, pp. 48-54, 2013.

[6] L. Menard and Y. Broise, "Theoretical and practical aspects of dynamic consolidation," Géotechnique, vol. 25, no. 1, pp. 3-18, 1975.

[7] W.-L. Zou, Z. Wang, and Z.-F. Yao, "Effect of dynamic compaction on placement of high-road embankment," Journal of Performance of Constructed Facilities, vol. 19, no. 4, pp. 316-323, 2005. 
[8] P. W. Mayne, J. S. Jones Jr., and J. C. Dumas, “Ground response to dynamic compaction," Journal of Geotechnical Engineering, vol. 110, no. 6, pp. 757-774, 1984.

[9] G. A. Leonards, W. A. Cutter, and R. D. Holtz, "Dynamic compaction of granular soils," Journal of the Geotechnical Engineering Division, vol. 106, no. 1, pp. 35-44, 1980.

[10] R. G. Lukas, "Dynamic compaction for highway construction, Design and construction guidelines, Volume I," Federal Highway Administration Report FHWA-RD-86-133, 1986.

[11] K. M. Rollins and J. Kim, "Dynamic compaction of collapsible soils based on U.S. case histories," Journal of Geotechnical and Geoenvironmental Engineering, vol. 136, no. 9, pp. 1178-1186, 2010.

[12] B. C. Slocombe, Dynamic Compaction. Ground Improvement, vol. 2, Glasgow, Chapman \& Hall. Cap., 1993.

[13] V. Luongo, "Dynamic compaction: predicting depth of improvement," Grouting, Soil Improvement and Geosynthetics, ASCE, pp. 27-939, 1992.

[14] Y. K. Chow, D. M. Yong, K. Y. Yong, and S. L. Lee, "Dynamic compaction of loose sand deposits," Soils and Foundations, vol. 32, no. 4, pp. 93-106, 1992.

[15] M. T. Smits and L. De Quelerij, "The effect of dynamic compaction on dry granular soils," in Proceedings of the International Conference on Soil Mechanics and Foundation Engineering, 12th, vol. 2, Rio de Janiero, Brazil, 1989.

[16] C. J. Poran and J. A. Rodriguez, "Finite element analysis of impact behavior of sand," Soils and Foundations, vol. 32, no. 4, pp. 68-80, 1992.

[17] F. H. Lee and Q. Gu, "Method for estimating dynamic compaction effect on sand," Journal of Geotechnical and Geoenvironmental Engineering, vol. 130, no. 2, pp. 139-152, 2004.

[18] K. F. Mostafa and R. Y. Liang, "Numerical modeling of dynamic compaction in cohesive soils," in Proceedings of the GeoFrontiers Congress 2011, pp. 738-747, Dallas, Texas, United States, 2011.

[19] M. Jia, Y. Yang, B. Liu, and S. Wu, "PFC/FLAC coupled simulation of dynamic compaction in granular soils," Granular Matter, vol. 32, no. 4, Article ID 76, 2018.

[20] J. Litwiniszyn, "The theories and model research of movements of ground masses," in Proceedings of the European Congress Ground Movement, pp. 203-209, Leeds, UK, 1957.

[21] B. C. Liu, "Ground surface movements due to underground excavation in the People's Republic of China," Excavation, Support and Monitoring, pp. 781-817, 1993.

[22] J. S. Yang, B. C. Liu, and M. C. Wang, "Modeling of tunnelinginduced ground surface movements using stochastic medium theory," Tunnelling and Underground Space Technology, vol. 19, no. 2, pp. 113-123, 2004.

[23] P. Guo, X. Gong, and Y. Wang, "Displacement and force analyses of braced structure of deep excavation considering unsymmetrical surcharge effect," Computers \& Geosciences, vol. 113, Article ID 103102, 2019.

[24] Y. Wang, S. Shan, C. Zhang, and P. Guo, "Seismic response of tunnel lining structure in a thick expansive soil stratum," Tunnelling and Underground Space Technology, vol. 88, pp. 250259, 2019.

[25] Y. P. Yao and B. Z. Zhang, "Reinforcement range of dynamic compaction based on volumetric strain," Rock and Soil Mechanics, vol. 9, Article ID 032, 2016 (Chinese).

[26] MH/T5035-2017, "Technical code for high filling engineering of airport," Civil Aviation Administration of China, 2017 (Chinese).
[27] A. Ghassemi, A. Pak, and H. Shahir, "A numerical tool for design of dynamic compaction treatment in dry and moist sands," Iranian Journal of Science \& Technology, Transaction B: Engineering, vol. 33, no. 4, pp. 313-326, 2009.

[28] A. Oshima and N. Takada, "Relation between compacted area and ram momentum by heavy tamping," in Proceedings of the International Conference on Soil Mechanics and Foundation Engineering-International Society for Soil Mechanics and Foundation Engineering, AA BALKEMA, vol. 3, pp. 1641-1644, 1997. 


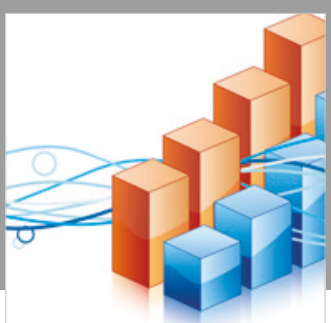

Advances in

Operations Research

\section{-n-m}
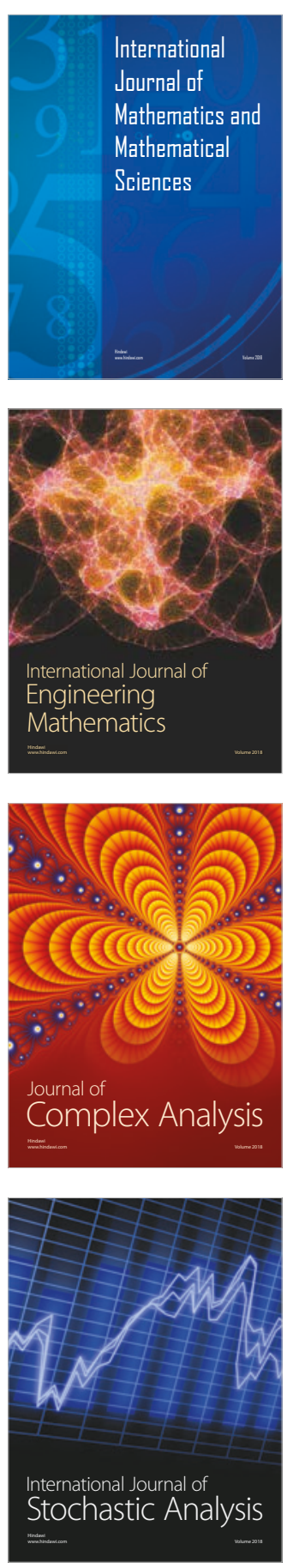
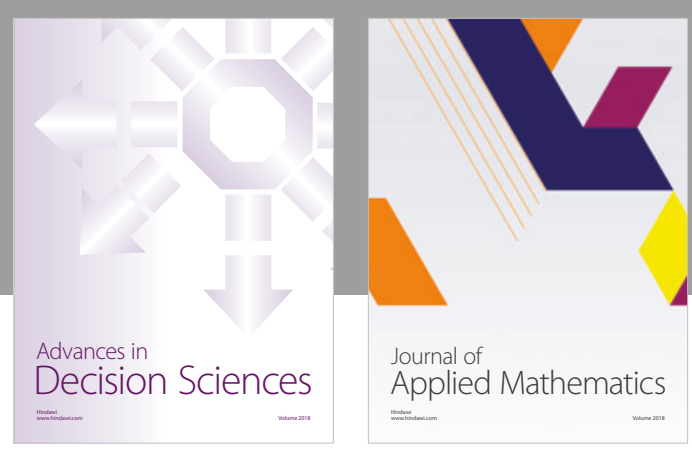

Journal of

Applied Mathematics
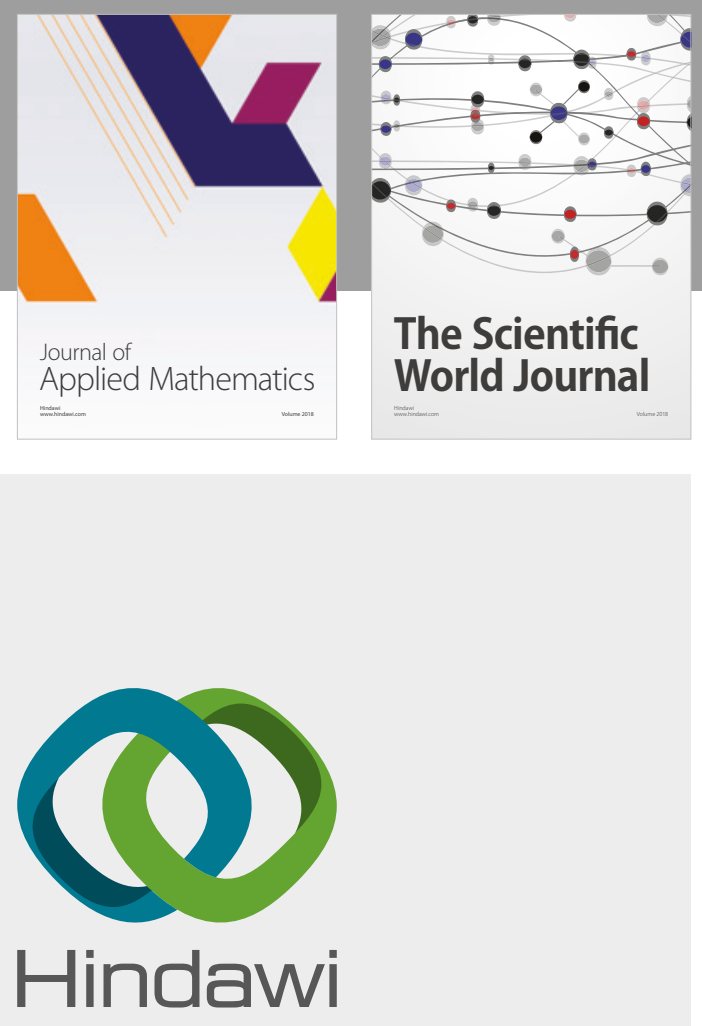

Submit your manuscripts at

www.hindawi.com

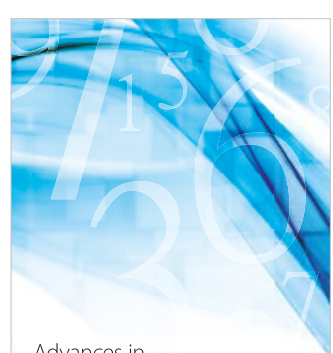

Advances in
Numerical Analysis
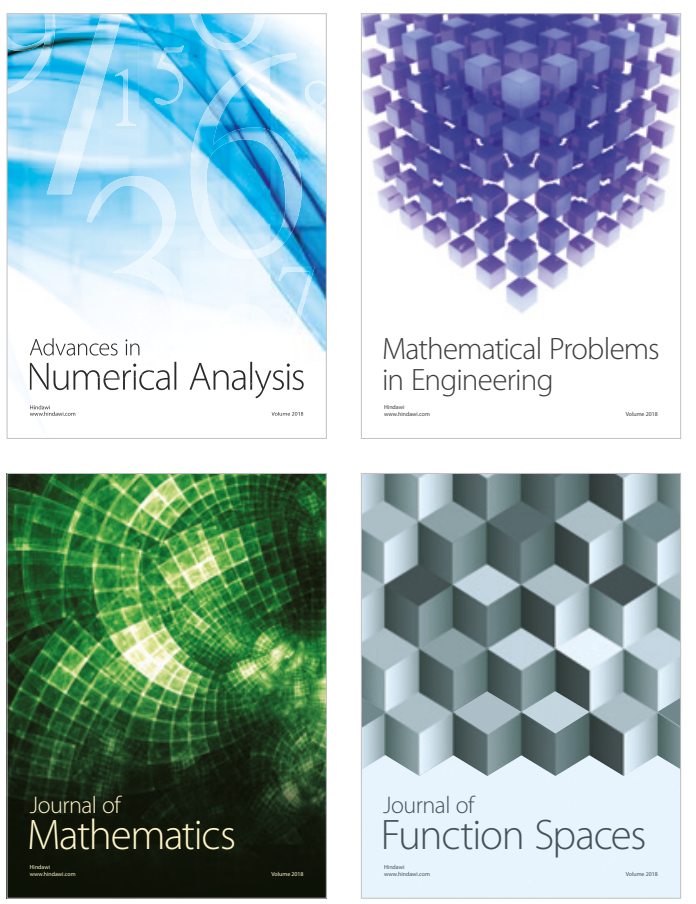

Mathematical Problems in Engineering

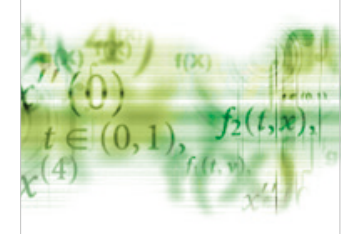

International Journal of

Differential Equations

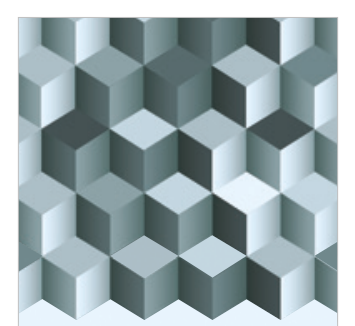

Journal of

Function Spaces
The Scientific

World Journal

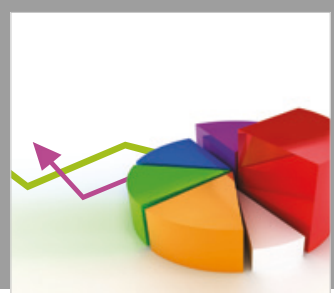

Journal of

Probability and Statistics
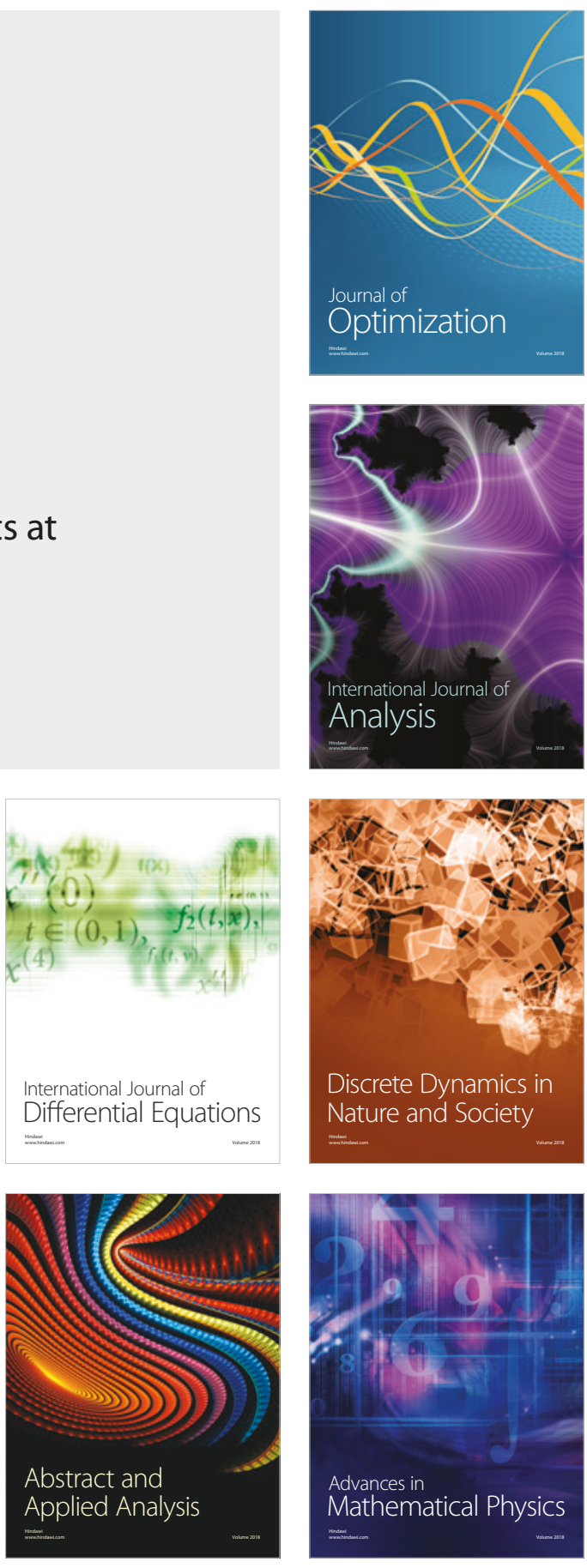